\title{
Crowd Behavior as an Example of the Evolution of a Complex System - Evacuation Models Proposal Based on the Symmetry Analysis Approach
}

\author{
W. SIKORA* AND J. MALINOWSKI \\ Faculty of Physics and Applied Computer Science, University of Science and Technology \\ al. A. Mickiewicza 30, 30-059 Kraków, Poland
}

\begin{abstract}
The evacuation of football stadium scenarios as examples of evolution of complex system are discussed. The models are presented as movements of individuals according to fields of displacements, calculated correspondingly to the given scenario. The assumption has been made that the most efficient evacuation is left based on the accordance of symmetry of allowed space, and this symmetry is taken into account while calculating the displacements field. The displacements related to every point of this space are calculated by the symmetry analysis method and fulfill the symmetry conditions of allowed space. The speed of each individual at every point in the presented model has the same quantity. Consequently, the times of evacuation and average presses acting on individuals during the evacuation are given. Both parameters are compared with and without symmetry considerations. They are calculated in the simulation procedure. For the realization of the simulation tasks the new program (using modified Helbing model) has been elaborated.
\end{abstract}

DOI: $10.12693 /$ APhysPolA.124.1005

PACS: $89.75 .-\mathrm{k}$

\section{Introduction}

The behavior of social systems influenced by some external conditions may be considered as analogical to the behavior of solid states exposed to temperature or external electric or magnetic fields. Both systems are complex, containing many interacting elements, and are realized in strictly defined spaces. These spaces are often strongly restricted, and, because of these restrictions, not all types of evolutions of these systems are allowed. When these spaces are symmetrical (i.e. in crystals) the types of systems' behavior, permitted by the symmetry of these spaces can be predicted on the basis of theory of groups and representations.

The symmetry analysis method (SAM) [1] has been successfully used for many years, allowing for the significant simplification of the descriptions of different types of phase transitions in crystals. The first attempt of the SAM application for modeling the evacuation of one floor of skyscraper, in which the symmetry of allowed space had been broken because of an accident, is presented in our work at knowledge-based and intelligent engineering systems - agents and multi-agent systems - technologies and applications (KESAMSTA) [2].

It has been proved that the evacuation along the paths related to the field of displacements calculated by SAM in accordance with symmetry of allowed space resulted in a slightly longer time of evacuation (because those paths are not the shortest ways to the exits) but the signifi-

*corresponding author; e-mail: sikora@novell.ftj.agh.edu.pl cantly lower average press acting on evacuated individuals, when compared with typical shortest paths.

It is known that too big press acting on evacuated persons during evacuation is likely to cause tragic accidents, so the possibility to lower this press seems to be important. Given the crowd psychology laws, it is hardly realistic to assume that in empty space of the floor the evacuated individuals would choose the SAM indicated paths to the exits and not the shortest way, even if they would be driven by the ambient intelligence (AMI) devices. There are some types of constructions, gathering many people, where there is the possibility to build the paths of evacuation in accordance with SAM field of displacements. The typical football stadium is an example of such a construction.

In this work the model of evacuation of one floor of skyscraper described in [2] is applied to one sector of the football stadium. The football stadium built from many similar sectors is a very good approximation of the structure fulfilling the periodic boundary conditions. The results of symmetry calculations done for the one floor of skyscraper may be applied also to planning the evacuation paths on the stadium sector. Below we discuss the evacuation of a stadium, whose architecture takes into account the symmetry calculations and compare it with evacuation of traditional stadium.

\section{Elements of the symmetry analysis method}

The symmetry group of an object is defined as special set of transformations which leave this object unchanged. Among the elements of space groups are translations, mirror planes, inversions and some special rotations around the axis, as well as combinations of them all. 
Translational symmetry gives the possibility to choose the small block named elementary cell. The repetition of it reconstructs whole system. In this way description of the whole system is reduced to description of elementary cell. Such procedure reduces the number of parameters needed to describe the system, but still this number may be decreased by using full symmetry restrictions, not only translational one. The properties of a given system in mathematical language are presented as functions defined on allowed space. In crystals those are for example: the displacements of atoms from initial equilibrium positions, the magnetic moments localized on given positions or probability of sites occupations. The transformation properties of such functions are given by matrix representations of allowed space symmetry group.

Theory of group representations had been applied many years ago for simplification of description of many-body, complex physical systems. Wigner, Lubarski, and Cracknell introduced (as "symmetric coordinates") the set of basis vectors of irreducible representations of molecules' symmetry groups in calculations of molecules vibrations. Lubarski discussed also the role of irreducible representations (IR) of crystal symmetry group in crystallographic second order phase transitions. This method was at first introduced to the description of magnetic ordering in crystals by Bertaut. Later that line of analysis has been developed by many other theoretists, like Izyumov and others [1]. The presentation of the functions, describing interesting system properties, localized on given set of symmetry equivalent positions, in the usually used frame of coordinates related to the edges of elementary cell (crystallographic system), as was mentioned previously, takes advantage of translation symmetry only. The other symmetry relations are abandoned in this description and as a consequence the description of many system properties is not as simple as it could be. It follows from rules of the theory of group representations, that any function defined in the space with given symmetry group may be presented as linear combination of basis vectors of irreducible representations (BVs) $\Psi$ of this group

$$
\boldsymbol{S}=\sum_{l, \nu, \lambda} c_{\lambda}^{\boldsymbol{k}_{l}, \nu} \Psi_{\lambda}^{\boldsymbol{k}_{l}, \nu}
$$

$l$ - number of $\boldsymbol{k}$ vectors describing the translational symmetry of $\approx S, \nu-$ number of IRs, $\lambda-$ number of dimensions of $\nu$ 's IR.

From Eq. (2.1) follows that the function $S$ may be treated as some vector in the functional space, where the BVs $\Psi$ play the same role in the presentation of function $S$, as axis of coordinate system, and coefficients $c_{\lambda}^{k_{l}, \nu}$ as components of it. When irreducible representation is taken into account, the dimension of this functional space invariant under all group transformations is the smallest one. It leads to the smallest number of $c_{\lambda}^{k_{l}, \nu}$ parameters, which in the frame of BVs $\Psi$ presents the function $S$. Each choice of these free parameters $c_{\lambda}^{k_{l}, \nu}$ uniquely determines one of the possible models of new structure that may be established after the phase transition. The presentation of model structures in the frame of basis vectors of irreducible representations of the initial symmetry group instead of that in the frame of crystallographic system $(x, y, z)$, is the best matching to the symmetry of the problem, as it provides the simplest (requiring the lowest number of independent parameters) form of the structure description.

The form of the basis vectors and the information which representations take part in the given phase transition are directly provided by the theory of groups and representations. It is important to note that the basis vectors have the same translational properties as the Bloch functions. Therefore, the basis vectors may be defined on positions in given elementary cell of the crystal as well as in the elementary cell translated by a lattice vector $\boldsymbol{t}$, which just corresponds to a multiplication by $\mathrm{e}^{\mathrm{i} k t}$. Not all the possible $c_{\lambda}^{k_{l}, \nu}$ are allowed, as the parameters should be selected in such a way that the resulting properties related to all atoms would have real values. This condition influences the set of equations which $c_{\lambda}^{k_{l}, \nu}$ have to satisfy and results in the reduction and strict determination of the number of independent free parameters. After the operation described above the final model contains clearly defined minimum number of free parameters and presents strictly defined relations between quantities describing considered property, localized on different crystal sites. The choice of representation $\tau_{\nu}$ and the coefficients $c_{\lambda}^{k_{l}, \nu}$ uniquely determines the symmetry of the new, ordered structure, regardless of the kind of the considered property. The type of phase transition and the property considered is included in the form of basis vectors. In the frame of SAM the discussion on connections between different types of transformations is also possible.

For the realization of the problems mentioned, the computer program MODY [3] based on the representations of symmetry space groups [1], and another associated programs - SPLIT, Tensor-vis, Tensor_OpenGL [4], have been elaborated. They are designed to find the all possibilities of new scalar, vector and tensor type structure properties allowed by the initial symmetry of the structure.

The discussion of the coexistence of different types of system behavior, leading to different properties of the system is based on the assumption, that different functions describing these properties of the system should have the same symmetry. It is already known from the theory of representation that these different functions should belong to the same irreducible representation of system symmetry group.

\section{Application the SAM to stadium evacuation scenario}

The scenario of the evacuation of football stadium organized according to SAM approach is discussed in this section and compared with the traditional model of sta- 
dium. By using the simulations with and without implementation of SAM results the time of evacuations and average press acting on the pedestrians during the evacuation are calculated.

The football stadium, similar as Allianz Arena in $\mathrm{Mu}-$ nich for example, is the space where the great number of individuals are localized, presenting (with quite good approximation) the translational symmetry with periodic boundary conditions (usually taken into account in crystals). This justifies discussing the evacuation of one stadium sector as the first step of designing the stadium evacuation scenario. It significantly reduces the number of evacuated individuals and, in consequence, the number of parameters used for scenario description.

The real symmetry of the sector is described by $P 1 m 1$ space group. The arrangement of chairs and evacuation paths guaranteeing quick and safe evacuation, the fields of displacement which correspond with full symmetry of available space (as polar vectors), and individuals speeds (as scalar quantities) should be calculated by using correspondingly the vector and scalar type SAM.

According to $P 1 m 1$ symmetry group the number of positions in the sector, which should be regarded is reduced to $1 / 2$, but the directions of displacements which should be defined on these positions remain free. Because the steps leading to exits, the displacements at each positions should have the $z$ components, directed to the upwards. As it is can be seen in Table, such relation between $z$ components belongs to the $\tau_{1}$ representation.
From SAM follows that $\tau_{1}$ does not change the initial symmetry and may be associated with any representation. The best choice of $x$ and $y$ components of displacement directions from evacuation point of view - as may be seen in Fig. 1 - can be made by treating the $P 1 m 1$ group as the broken symmetry of $P m m 2$ group generated by $\tau_{4}$ representation of $\boldsymbol{k}=(0,0,0)$. This leads to field of displacements, and corresponding field of speeds as given in Table quoted below. Taking into account the vector field of displacements got by the treatment mentioned above the paths for evacuation are designed in one stadium sector.

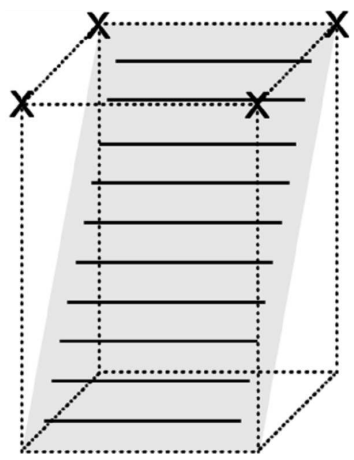

Fig. 1. The restriction of allowed space from $P m m 2$ to $P 1 m 1$ symmetry subsequent with installation of chairs in the stadium sector.

The results of SAM vector and scalar type calculations applied to stadium sector.

TABLE

\begin{tabular}{|c|c|c|c|c|c|c|}
\hline \multirow{3}{*}{$\begin{array}{c}\text { Representation } \\
\text { and } \\
\text { destination } \\
\text { group }\end{array}$} & \multirow{3}{*}{ Version } & \multicolumn{5}{|c|}{$\begin{array}{l}\text { The polar vector type and scalar type basic vector functions of representations of } P m m 2 \\
\text { space group with } k=(0,0,0) \text {, defined at positions } 4 \mathrm{i} \text { in the elementary cell }\end{array}$} \\
\hline & & \multirow{2}{*}{ Free parameter } & \multicolumn{4}{|c|}{ Pos. 4i } \\
\hline & & & 1: $(x, y, 0)$ & $2:(1-x, 1-y, 0)$ & $3:(1-x, y, 0)$ & $4:(x, 1-y, 0)$ \\
\hline \multirow{4}{*}{$\begin{array}{c}\text { Tau1 } \\
\text { destination } \\
\text { group } \\
\text { Pmm2 }\end{array}$} & vectorI & $A_{1}$ & $(1,0,0)$ & $(-1,0,0)$ & $(-1,0,0)$ & $(1,0,0)$ \\
\hline & vectorII & $B_{1}$ & $(0,1,0)$ & $(0,-1,0)$ & $(0,1,0)$ & $(0,-1,0)$ \\
\hline & vectorIII & $C_{1}$ & $(0,0,1)$ & $(0,0,1)$ & $(0,0,1)$ & $(0,0,1)$ \\
\hline & scalar & $P 1$ & 1 & 1 & 1 & 1 \\
\hline \multirow{4}{*}{$\begin{array}{c}\text { Tau2 } \\
\text { destination } \\
\text { group } \\
P 112\end{array}$} & vectorI & $A_{2}$ & $(1,0,0)$ & $(-1,0,0)$ & $(1,0,0)$ & $(-1,0,0)$ \\
\hline & vectorII & $B_{2}$ & $(0,1,0)$ & $(0,-1,0)$ & $(0,-1,0)$ & $(0,1,0)$ \\
\hline & vectorIII & $C_{2}$ & $(0,0,1)$ & $(0,0,1)$ & $(0,0,-1)$ & $(0,0,-1)$ \\
\hline & scalar & $P 2$ & 1 & 1 & -1 & -1 \\
\hline \multirow{4}{*}{$\begin{array}{c}\text { Tau3 } \\
\text { destination } \\
\text { group } \\
\text { Pm11 }\end{array}$} & vectorI & $A_{3}$ & $(1,0,0)$ & $(1,0,0)$ & $(-1,0,0)$ & $(-1,0,0)$ \\
\hline & vectorII & $B_{3}$ & $(0,1,0)$ & $(0,1,0)$ & $(0,1,0)$ & $(0,1,0)$ \\
\hline & vectorIII & $C_{3}$ & $(0,0,1)$ & $(0,0,-1)$ & $(0,0,1)$ & $(0,0,-1)$ \\
\hline & scalar & $P 3$ & 1 & -1 & 1 & -1 \\
\hline \multirow{4}{*}{$\begin{array}{c}\text { Tau4 } \\
\text { destination } \\
\text { group } \\
P 1 m 1\end{array}$} & vectorI & $A_{4}$ & $(1,0,0)$ & $(1,0,0)$ & $(1,0,0)$ & $(1,0,0)$ \\
\hline & vectorII & $B_{4}$ & $(0,1,0)$ & $(0,1,0)$ & $(0,-1,0)$ & $(0,-1,0)$ \\
\hline & vectorIII & $C_{4}$ & $(0,0,1)$ & $(0,0,-1)$ & $(0,0,-1)$ & $(0,0,1)$ \\
\hline & scalar & $P 4$ & 1 & -1 & -1 & 1 \\
\hline
\end{tabular}

\section{Helbing social force model modifications for real-time calculations.}

It is extremely difficult to conduct the experiment of evacuation of crowd from an existing stadium. The con- struction of new, nonstandard architecture of sector just for the experiment is impossible. In such situation the most efficient method of scientific investigations is computer simulation. Currently a few different methods of 
crowd movement simulation are used, which can be divided in two types. One is based on discrete cellular automata and the other is based on continuous so-called social force originally presented by Helbing [5]. The methods of the first kind are quick and give the possibility to simulate the movement of a big number of individuals in complicated geometry, but - as discrete - they are far from real behavior and give no possibility to calculate the press acting on evacuated pedestrians. The methods of the second kind work in continuous space and allow for calculations of acting press, but they are time consuming (however they can be performed even in real-time for small numbers of pedestrians [6]).

In this paper we present the results of simulations realized using modified Helbing method elaborated by Malinowski. The aim of this method is presented below.

The original Helbing model $[5,7]$ is based on the equation of motion (4.1) of the $i$-th individual

$$
m_{i} \frac{\mathrm{d} \boldsymbol{v}_{i}}{\mathrm{~d} t}=m_{i} \frac{v_{i}^{0} \boldsymbol{e}_{i}^{0}(t)-\boldsymbol{v}_{i}(t)}{\tau}+\sum_{j(\neq i)} \boldsymbol{f}_{i j}+\sum_{W} \boldsymbol{f}_{\mathrm{i} W}
$$

$\boldsymbol{f}_{i}^{\mathrm{D}}=m_{i}\left[v_{i}^{0} \boldsymbol{e}_{i}^{0}(t)-\boldsymbol{v}_{i}(t)\right] / \tau$ is the driving force responsible for keeping desired velocity, $\boldsymbol{f}_{i j}$ is an interaction force induced by other pedestrian, given by some continuous potential function, $\boldsymbol{f}_{\mathrm{i} W}$ is an interaction force between pedestrian and walls or other obstacles also given by some continuous potential function.

For many moving individuals and dynamically changing situation the simulation requires powerful computer facilities to run in real time. The modified model should fulfill such advantages of the Helbing original model as realistic movement in continuous space, but the program based on this model will use only small part of computational power needed to run original Helbing model. Then it will provide the possibility of introducing at last some of the Helbing model's exceptional advantages into small portable devices (such as cell phone).

First changes are made in the first part of the sum on the right side of the equation. " $\boldsymbol{e}_{i}$ " is a vector that shows the desired "evacuation velocity" direction. As we have shown before - in our previous implementation of social force model [2] - this vector can be precalculated and stored in array to increase simulation speed. Precalculation of thoses desired velocities is divided into two steps. First step uses well known weave-front method [6, 8, 9]:

1) divide space into cells and mark exit cells with " 0 ", obstacles with " -2 " and free space with " -1 ";

2) for all cells labeled "-1" check for minimal number (greater or equal " 0 ") in all adjacent cell, label this cell with founded value +1 ;

$3)$ continue point 2 as long as cells with value "-1" exists. As the result we get the field of numbers (Fig. 2a).

In the second step we cast lines from each cell in every direction and look for minimal value. The length of the normalized vector that connects the actual cell with the cell with the lowest founded value (in this case " 4 ") is the desired direction (as shows in Fig. 2b). At least the field of displacements is got (Fig. 2c).

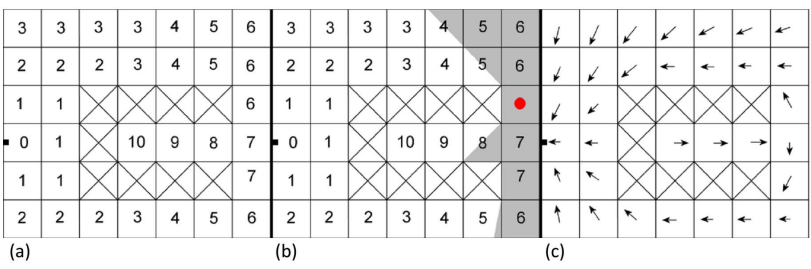

Fig. 2. Three steps to precalculate $\boldsymbol{e}_{i}$ : (a) calculating "distance" to exit for each cell, (b) "ray-casting" from each cell to found best direction for that cell, (c) final result - each cell has preferred moving direction $\boldsymbol{e}_{i}$.

The second part of the sum in (4.1) is responsible for pedestrian-pedestrian interaction. Pedestrian can get tightened a little when pushed by the other pedestrians. On the one hand, this concept allows to predict what happens with pedestrians in panic situation when everyone rushes to the exit, but on the other hand calculating this sum is a time consuming task. In our simplified model we decide not to calculate this force at all. This part was replaced by circle-circle collision detection algorithm. Detecting collision between two moving circles can be done very fast - for example using van den Heuvel method (as presented in "Gamasutra") [10]. We lose this way the possibility of the "per pedestrian" force calculation.

When a pedestrian wishes to move in some direction and the other pedestrian stays on his/her way - he/she simply cannot move forward - exactly like in the Helbing model. The advantage of the standard Helbing model in that situation is that the pedestrian in fact "pushes" other pedestrians and thus generates an additional force on all the pedestrians that stay in front of him/her. In our model this is not the case - we sacrified here the quality of simulation for its speed.

The last part of a sum is pedestrian-obstacle force calculation. This part contains also a small force that reflects the fact that in most cases people do not want to stay too close to wall. In our case we took only these small forces into consideration, and we pre-calculated it for "average sized pedestrian" and added them to previously calculated field of forces. We lost another nice feature of original Helbing model here - when pedestrian stays close to wall and other pedestrians push him/ her, the original model allows to calculate how large force is applied to this pedestrian - and when it exceeds some value we can consider pedestrian seriously injured. In our model we assume that all obstacles are constructed from segments and we use fast line-circle collision detection algorithm to avoid pedestrians walking through walls [11].

If no real time calculations are required, the new future can be reintroduced into the model. During the lecture hall H4 experiment made on AGH it became obvious that the "shortest path approach" to simulation is not the best 
option. As it has been previously described we use "front weave method" to find the shortest path. In such an approach pedestrians starting from point 1,2 , and 3 go to the left (Fig. 3a), is there anyone sitting in the same row or not.

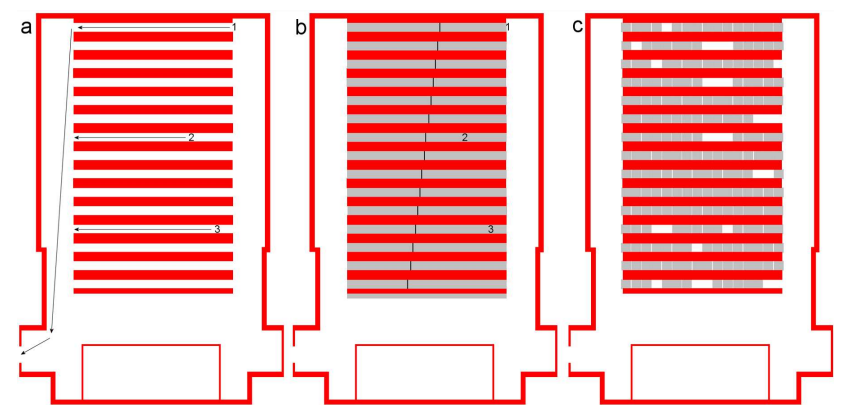

Fig. 3. Changes made in shortest part algorithm to better mirror reality. (a) Original algorithm. Agents 1,2 and 3 move to the left no matter how many agents "sit" in every row. (b) Adding some "penalty" areas between rows. This areas are independent of pedestrians density. (c) Additional penalty areas based on actual pedestrian position.

We introduce combination of two solutions to eliminate such an unnatural pedestrians' behavior. The first solution does not depend of the starting arrangement of pedestrians. When we look at the $\mathrm{H} 4$ hall plan we can notice that pedestrians may consider space between desks not so comfortable to walk - thus they may prefer to walk to the right side where more comfortable walk area is located. To incorporate that fact into our simulation we introduced the so-called "penalty areas" (grey areas in Fig. 3b).

In the white area the front weave method takes distance between neighbor cells equal to 1 . In the grey area the front weave method changes cell to cell value by more than 1 (1.6 in our case). This change in algorithm divides the rows into parts, that are not equal. The pedestrians that found themselves at the start on the right side of the black line "decide" to go to the right whereas the pedestrians that found themselves on the left side of the black line "decide" to go to the left. Adding this feature makes our simulation a little more realistic but still far from the real experiment.

The second solution depends on pedestrians' location. It can be used as both static and dynamic method. In the static case (we use this approach in our case) more penalties area are added in preparation phase when "desired velocities" are calculated. In every place where pedestrians are present at the beginning of simulation we place additional penalty area in the shape of square with size $a=3 r$ (where $r$ is a radius of pedestrian) (Fig. 3c).

After adding this extra area, the simulation almost precisely mimics reality. Some pedestrians choose left side when the others choose the right side. In the dynamic version of those "penalty areas" from pedestrians can be applied in every simulation step. We did not test this solution due to some efficiency problem.

As the information about the press acting on pedestrian is very important, in the next step of our simplified model the magnitude of force acting on " $i$ "-th agent from other agents (pressing on given agent) is calculated according to formula

$$
F_{i^{p}}=\sum_{j \neq i} \frac{{ }_{j}\left(\boldsymbol{r}_{i}-\boldsymbol{r}_{j}\right) \cdot \boldsymbol{e}_{j}}{\left|\boldsymbol{r}_{i}-\boldsymbol{r}_{j}\right|} .
$$

The sum is going over $j \neq i$ and $j \in\left\{n^{\prime}\right\}$ - the set of agents, which are at given moment $t$ in state of collision with some other agent, $A$ is some constant (related for example to emotional states of agents, their speeds etc.), $\left|\boldsymbol{r}_{i}-\boldsymbol{r}_{j}\right|$ is the corresponding distance between $i$ and $j$ agents, $\boldsymbol{e}_{j}$ are the unit vectors along the directions of motion of $j$-th agents. Force transfer in this model is similar to the one of a system of hard spheres. Such treatment gives, even in our simplified model, the possibility to analyze approximate quantity of average force (pressing) acting on a pedestrian during evacuation, maximal and minimal per-pedestrian pressing and places where they appear.

\section{Simulation of stadium evacuation}

The vector field calculated by SAM for a stadium sector is presented in Fig. 4. The sector architecture which

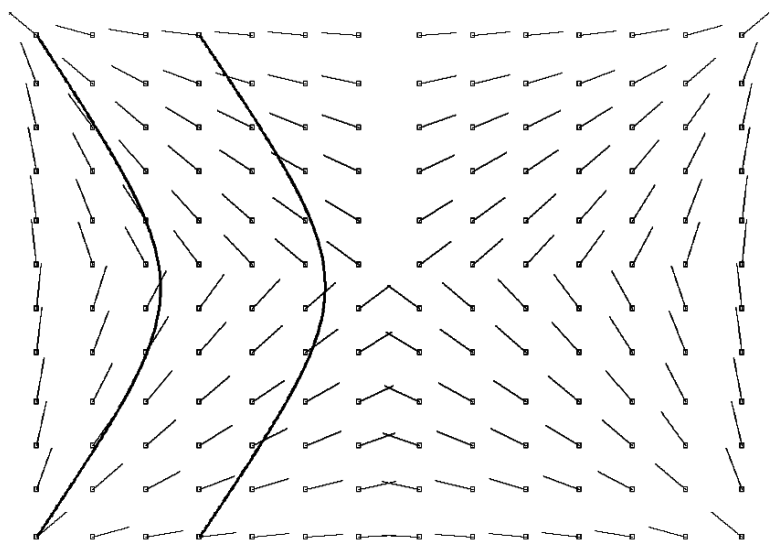

Fig. 4. The vector field calculated by SAM related to one stadium sector for $P m m 2$ space group and $\tau_{4}$ representation of $\boldsymbol{k}=(0,0,0)$.

corresponds with SAM calculations in outlining the evacuation paths is presented in Fig. 5, which gives the map of free spaces, obstacles and exits needed for calculation of forces acting on individuals as mentioned in Eq. (2) and velocities field used in the simulation (Fig. 6). The traditional stadium sector architecture is given in Fig. 7 .

On the basis of SAM model four different cases with some closed paths, where the pedestrian have only one possibility to move to the exit and some open paths, where their choice is free, has been proposed in comparison with "normal" sector case. Figure 8 shows all four types of sectors proposed. 


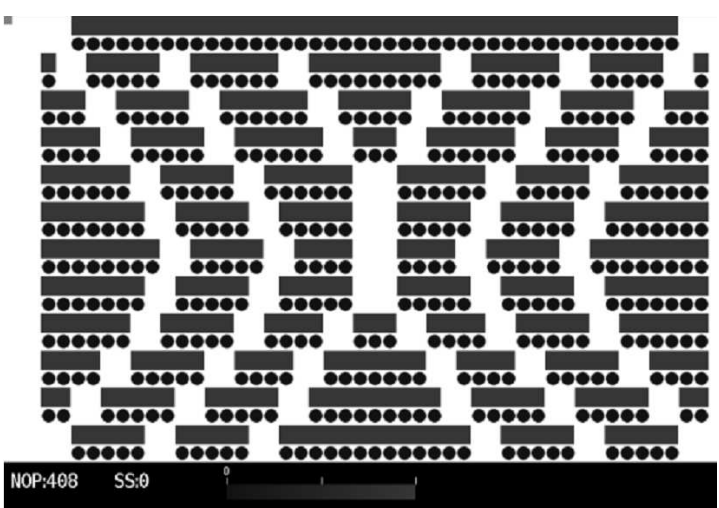

Fig. 5. Construction of the evacuation paths in a stadium sector corresponding with SAM vector field calculations.

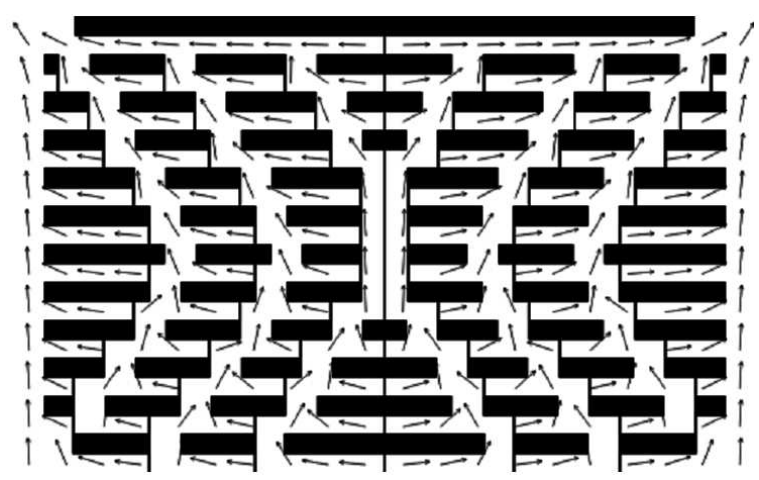

Fig. 6. Table of the final field of velocities calculated for the simulation of a stadium sector evacuation for SAM model.

For all of this sector types the time of evacuation and press per pedestrian during evacuation has been calculated twofold: for two exits open (Fig. 9a and b) and for one exit open (10a and b).

The differences between normal and symmetrical sector (case $d$ ) when the press per pedestrian is considered

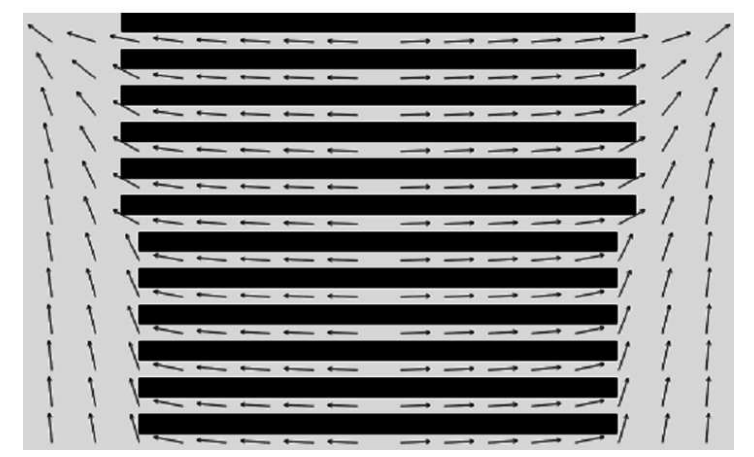

Fig. 7. Table of the final field of velocities calculated for the simulation of a stadium sector evacuation for traditional model.

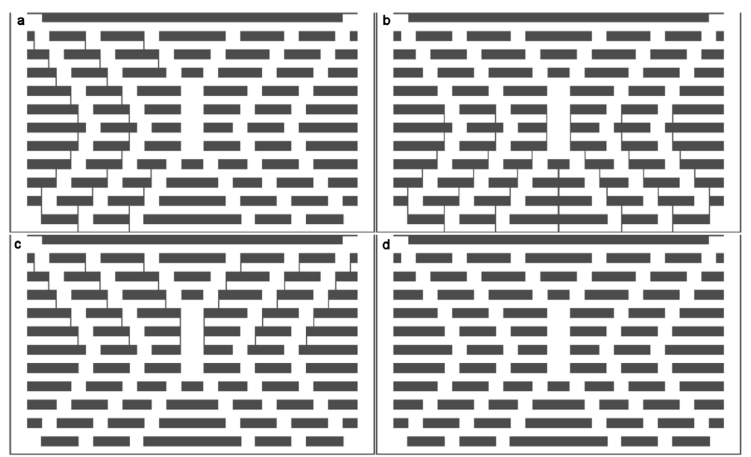

Fig. 8. Different types of symmetry related stadium sectors considered in this work.

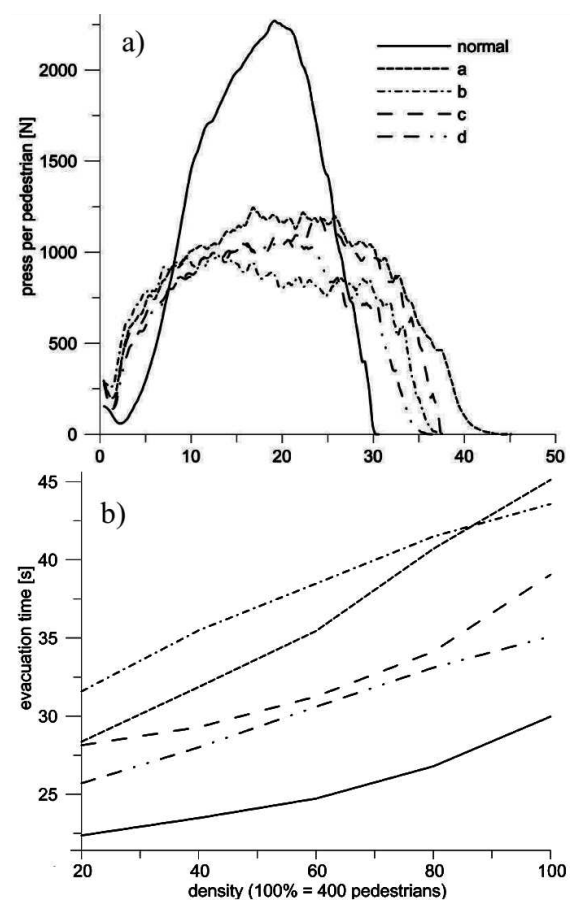

Fig. 9. (a) Press per pedestrian in function of time. Two exits open. (b) Total evacuation time in function of density. Two exits open.

in function of time and the initial density of pedestrians is given in Figs. 11 and 12. The functions presented in Fig. 11 are calculated for two exits opened, while in Fig. 12 are for one exit opened.

The time of evacuation and average press acting on the individuals are calculated during the simulation by using SAM related and traditional architecture of stadium. The number of evacuated individuals - $408-$ taken for simulation corresponds with number of sites in the sector of Allianz rena stadium and is the same for both analyzed architecture types. These two models differ only in distribution of chairs and evacuation paths layout inside the sector. The results for both models $\mathrm{SAM}$ related and traditional — are given as the plots 


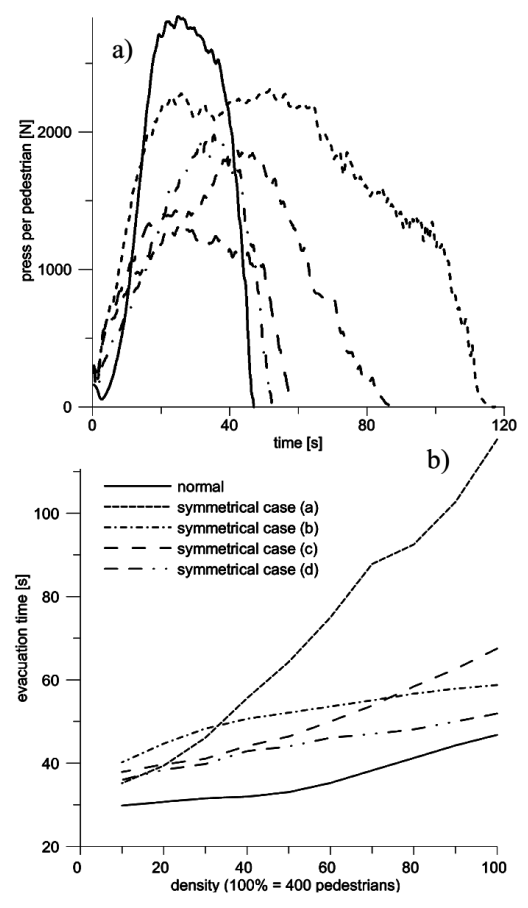

Fig. 10. (a) Press per pedestrian in function of time. One exit open. (b) Total evacuation time in function of density. One exit open.

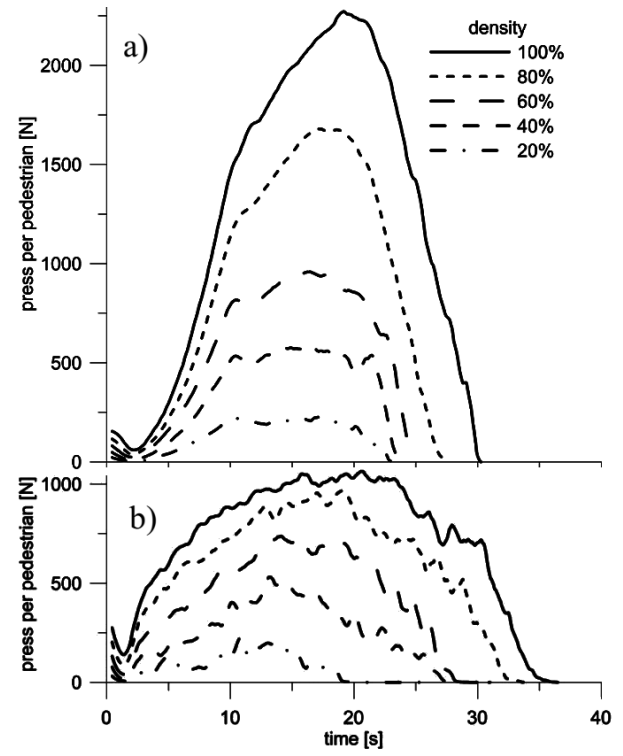

Fig. 11. (a) Normal sector. Press per pedestrian in function of time. Two exits open. (b) Symmetrical sector. Press per pedestrian in function of time. Two exits open.

presenting the average press acting on one individual as the function of time from the beginning to the end of evacuation (Figs. 9-11).

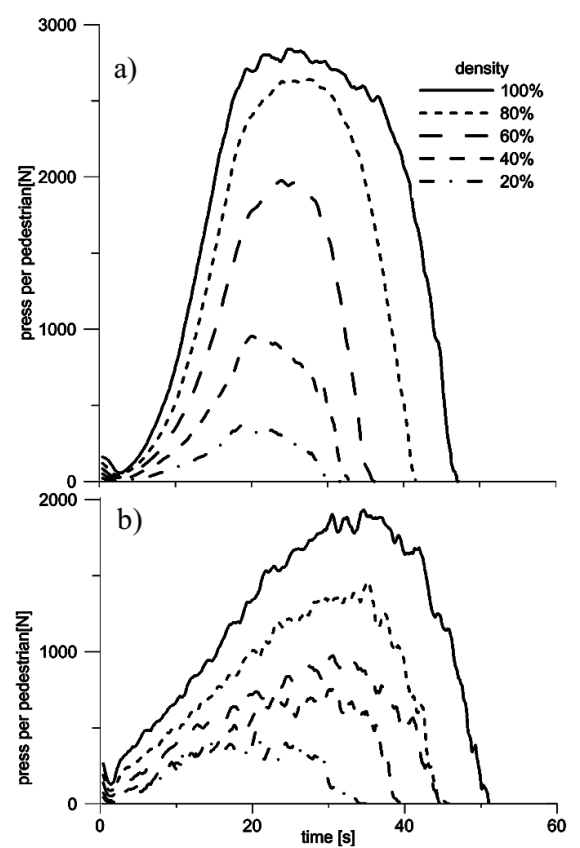

Fig. 12. (a) Normal sector. Press per pedestrian in function of time. One exit open. (b) Symmetrical sector. Press per pedestrian in function of time. One exits open.

\section{Conclusions}

A new model of football stadium sector's architecture, leading to the most efficient sector evacuation is proposed. As the criterion of evacuation efficiency the two parameters - the time of evacuation $t_{\mathrm{e}}$ (expressed by the steps of simulation) and the average press acting on the individuals during the evacuation $F_{\mathrm{a}}$ - have been considered. Within the model, in which the evacuation paths are related to stadium symmetry, as may be seen in the presented figures, the press $F_{\mathrm{a}}$ is significantly limited while the $t_{\mathrm{e}}$ remains almost the same as for the traditional construction. The same solution, taking into account the symmetry of the allowed space can be used in designing the evacuation routes for the mass open-air events, gathering thousands of peoples, such as concerts, rallies, religious gatherings, etc.

For the purpose of the task presented in this article the new program has been developed. Several modifications of standard Helbing model has been presented. Some changes in original Helbing model, e.g. preferred moving direction precalculation, lead to the simplification of calculations. Other changes - e.g. "penalty areas" — lead to the more realistic estimation of agents' behavior.

\section{Acknowledgments}

This work is partially supported by EU program SOCIONICAL (FP7 No. 231288). 


\section{References}

[1] W. Sikora, L. Pytlik, in: Group Theory: Classes, Representations and Connections, and Applications, Nova Sci. Publ., New York 2010, Ch. 1, p. 1.

[2] W. Sikora, J. Malinowski, in: 4th KES Int. Symp., KES- MST 2010, Gdynia (Poland), 2010, Proc. Part II, Lecture Notes in Artificial Intelligence, Subseries of Lecture Notes in Computer Science, Eds. R. Goebel, J. Siekmann, W. Wahlster, Springer-Verlag, Berlin 2010, p. 229.

[3] W. Sikora, F. Białas, L. Pytlik, J. Appl. Crystallogr. 37, 1015 (2004). Program available from: http:// novell.ftj.agh.edu.pl/sikora/mody.htm .

[4] Tensor Vis program available at: http: //novell.ftj.agh.edu.pl/ malinowski/opis/ Tensor_Vis_en.html.

[5] D. Helbing, I. Farkas, T. Vicsek, Nature 407, 487 (2000).
[6] N. Courty, S. Raupp Musse, in: CGI'05 Proc. Computer Graphics Int. 2005, p. 206.

[7] D. Helbing, P. Molnár, Phys. Rev. E 514282 (1995).

[8] T. Kretz, C. Bonish, P. Vortish, in: Pedestrian and Evacuation Dynamics 2008, Eds. W.W.F. Klingsch, Ch. Rogsch,A. Schadschneider, M. Schreckenberg, Springer, Heidelberg 2010, p. 335.

[9] W. Wolf, Modern VLSI Design, Prentice Hall PTR, Ann Arbor 1998, p. 518.

[10] http://www.gamasutra.com/view/feature/131424/ pool_hall_lessons_fast_accurate_.php .

[11] C. Ericson, Real Time Collision Detection, Elsevier/Morgan Kaufmann Publishers, San Francisco 2005 . 